\title{
Ultrafast single electron spin manipulation in 2D semiconductor quantum dots with optimally controlled time-dependent electric fields through spin-orbit coupling
}

\author{
J. A. Budagosky \\ Institute for Biocomputation and Physics of Complex Systems, \\ University of Zaragoza, Mariano Esquillor s/n, 50018 Zaragoza (Spain) \\ A. Castro \\ ARAID Foundation, Edificion CEEI, \\ María Luna 11, 50018 Zaragoza (Spain) and \\ Institute for Biocomputation and Physics of Complex Systems, \\ University of Zaragoza, Mariano Esquillor s/n, 50018 Zaragoza (Spain)
}

(Dated: March 25, 2017)

\begin{abstract}
We have studied theoretically the possibility of ultra-fast manipulation of a single electron spin in 2D semiconductor quantum dots, by means of high-frequency time-dependent electric fields. The electron spin degree of freedom is excited through spin-orbit coupling, and the procedure may be enhanced by the presence of a static magnetic field. We use quantum optimal control theory to tailor the temporal profile of the electric field in order to achieve the most effective manipulation. The scheme predicts significant control over spin operations in times of the order of picoseconds an ultrafast time scale that permits to avoid the effects of decoherence if this scheme is to be used as a tool for quantum information processing.
\end{abstract}




\section{INTRODUCTION}

Quantum dots are nanoscopic artificial structures, somehow created within solid state devices, that contain a small number of charge carriers (electrons or holes), and display quantum behaviour in the same manner that atoms or molecules do [1]. Nowadays it is relatively easy to isolate even one single electron. The precise control over charge and currents on quantum dots was soon achieved and demonstrated, whereas the experimental techniques to measure and manipulate the spins followed later on [2]. These developments have contributed to the growth of the field of spintronics [3], which consists of the control and manipulation of the spin degrees of freedom in solid state devices.

Among the many foreseen applications of quantum dot based spintronics is quantum information processing: the spin of a single electron is the most typical example of a twolevel system, and this fact soon suggested the possibility of using single-electron quantum dots as a physical realization of a qubit [4]. One of the reasons for this to be conceivable is the long decoherence times observed for the spin degree of freedom in common quantum dots $[5,6]$ (other reason is the realization, at least at the level of proof-of-principle, of the DeVincenzo's criteria [7]). This decoherence time is to be compared with the time required for an operation, i.e. the typical time used to change the state of the system in a controlled manner, with an external field. It is therefore essential to have a means to produce very fast operation times, specially since fault-tolerant operations require multiple possible manipulations within the coherence time.

In order to induce a spin flip in these systems, one can of course use a time-dependent magnetic field, oscillating at the Zeeman transition frequency; the Rabi oscillations will eventually induce full transitions from one state to another. This procedure is called "electron spin resonance" (ESR). However, this method is not particularly fast, and moreover it is not easy to produce and localize these magnetic fields individually on each quantum dot. Recently, the alternative use of electric fields has also been proposed and demonstrated [812], since these can indirectly couple to the spin, for example through spin-orbit coupling. An electric field may be produced locally on chip through appropriate gates, or one may attempt optical manipulation via a $\mathrm{THz}$ laser pulse, which has the advantage of a very high frequency, and therefore promise very fast spin rotations.

In this work we will focus on this second option, the optical manipulation of spins - 
or, in any case, the use of high frequency electric fields, whatever its origin. The action of $\mathrm{THz}$ optical fields on quantum dots, and its coupling to the spin degree of freedom thorough spin-orbit coupling, has already been theoretically investigated for example by Jiang et al. [13] In this work, we inquire into how fast can a single spin in a quantum dot rotate when manipulated with a $\mathrm{THz}$ electric field, through the indirect coupling facilitated by the spin-orbit interaction. We will not limit the allowed external fields to quasi-monochromatic laser pulses (that are typically tuned to some resonant frequency), but rather consider the possibility of shaping the temporal shape of the pulse, in order to find a path that produces the transition in a faster way than following Rabi's oscillations. In order to find the shape that produces the optimal result, we will use quantum optimal control theory (QOCT) $[14,15]$. This theory provides a set of techniques to find the best external fields that, acting on a quantum system, produce the evolution that is optimal in a certain sense defined by the user.

We have implemented QOCT in the real-space, real-time, electronic structure code octopus [16-18]. This code focuses on the time-dependent many-electron problem, based on time-dependent density-functional theory [19, 20], although in this case this feature is not necessary since we deal with a single-electron problem. This implementation of QOCT has already been employed in recent years to study dynamics of $2 \mathrm{D}$ quantum dots and rings in the presence of $\mathrm{THz}$ laser fields [21-23]. By including in the model a spin-orbit coupling term (in this case, we have chosen the Rashba term [24], although this choice is not important for the conclusions that follow), we have learned what are the typical transition velocities that one may expect when manipulating the spin of a single electron quantum dot with this kind of electric fields.

The goal has been to construct optimal laser pulses that drive a single-electron spin from a given initial orientation in the Bloch sphere to any other target spin orientation. Within QOCT, such physical goals have to be mathematically formalized with the definition of a "target function" that determines the degree of success achieved for the task that is pursued. In this work we have considered two possible target definitions: The first one corresponds to the projection into some pre-defined spin orientation: the control is exerted on the orientation of spin without specifying a priori which stationary states are involved. The second target corresponds to the transition from the ground state, which is known to have a dominant spin in one particular direction, to an excited eigenstate that has a 
dominant spin in an approximately opposite direction.

\section{THEORY}

We consider the quantum dots fabricated on top of the two-dimensional electron gas (2DEG) that can be locked at the interface of a semiconductor heterostructure. The most common case is perhaps that of the GaAs/AlGaAs heterostructure, and therefore we will consider this material in the following. The AlGaAs layer is usually doped with Si, which results in the liberation of free electrons, that accumulate at the GaAs/AlGaAs interface, and are trapped in a thin (around $10 \mathrm{~nm}$ ) layer. The electronic system can then be considered to occupy a thin potential well in the direction perpendicular to the interface plane (hereafter, the $z$-direction). This thinness (to be compared to the Fermi wave length of the electrons, large due to the low electronic density) is the reason for the $2 \mathrm{D}$ character of the system, as the electrons can be considered to occupy only the lowest subband - at the low temperature that are necessary for these experiments to take place. Once the 2DEG is thus created, one may further constrain electrons in the $x y$ plane, by placing gates (metal electrodes) on top of the semiconductor, and controlling their voltages.

The electronic islands created in this manner (the quantum dots) can then be modeled by considering an effective mass approximation in 2D, and assuming simple and smooth confining potentials in the $x y$ plane - typically, as we will do below, of parabolic form. In addition, one may have an external magnetic field, a time-dependent external electric field (originated by the variation of the potential applied on the gates, or by a laser source), and, as we will crucially consider in this work, one or more spin-orbit coupling (SOC) terms.

There are various forms of SOC that can be present in this kind of zincblende materials. The bulk crystal lacks inversion symmetry, which leads to the Dresselhaus term [25], and in addition the heterojunction produces a structural inversion asymmetry along the growth direction that results in the Rashba term [24]. The strength of the Rashba effect can in fact be tailored with the application of external electric fields applied in parallel to the growth direction. Because of this tunability, we have chosen to work exclusively with this Rashba term, which is in many circumstances the dominant one [26, 27]. However, the qualitative conclusions that we will draw out do not depend on this choice: these SOC terms couple to the external electric fields in a similar manner. 
After all these considerations, the system can be modeled, in the absence of external time-dependent electric pulses, with the following static effective Hamiltonian:

$$
\hat{H}_{0}=\frac{\hbar^{2}}{2 m^{*}}\left(-i \boldsymbol{\nabla}-\frac{e}{\hbar} \boldsymbol{A}(\hat{\boldsymbol{r}})\right)^{2}+V_{c}(\hat{\boldsymbol{r}})+\alpha\left[\hat{\boldsymbol{\sigma}} \times\left(-i \boldsymbol{\nabla}-\frac{e}{\hbar} \boldsymbol{A}(\hat{\boldsymbol{r}})\right)\right]_{z}+\frac{g^{*}}{2} \mu_{B}(\hat{\boldsymbol{\sigma}} \times \boldsymbol{B})_{z}
$$

The first term corresponds to the electron kinetic energy, where $m^{*}$ is the electron effective mass that we consider to be $m^{*}=0.067 m_{e}$ in a GaAs semiconductor medium $\left(m_{e}\right.$ is the electron mass). The vector potential $\boldsymbol{A}(\hat{\boldsymbol{r}})$ included in that term generates the static homogeneous magnetic field $\boldsymbol{B}=B \boldsymbol{z}$, normal to the $x y$-plane where the system is confined. The second term,

$$
V_{c}(\hat{\boldsymbol{r}})=\frac{1}{2} m^{*} \hbar \omega_{0}\left(\hat{x}^{2}+\hat{y}^{2}\right)
$$

is the confinement potential. The third term correspond to the Rashba SOC, whereas the fourth is the Zeeman term. In those expressions, $\hat{\boldsymbol{\sigma}}=\left(\hat{\sigma}_{x}, \hat{\sigma}_{y}, \hat{\sigma}_{z}\right)$ is the vector of Pauli matrices, $\alpha$ is the Rashba parameter that determines the SOC strength, $g^{*}$ is the effective gyromagnetic factor (we will use $g^{*}=-0.44$ for GaAs), and $\mu_{B}$ is the Bohr magneton.

For the confinement potential, we have used $\hbar \omega_{0}=1.8 \mathrm{meV}$, which is in the range of the typical values in common experimental realizations of QDs. A rough corresponding estimate of the QD lateral extension is approximately $2 \sqrt{\hbar / m^{*} \omega_{0}} \approx 50 \mathrm{~nm}$, which also lies within the typical range of sizes for lithographically etched and gate-confined QDs. In the following, we will express all the quantities in effective atomic units, that relate to usual atomic units (defined by setting $e^{2}=m_{e}=\hbar=1$ ) in the following manner: $a_{0}^{*}=a_{0}\left(m^{*} / \kappa\right)$, $H_{a}^{*}=H_{a}\left(\kappa^{2} / m^{*}\right), t_{0}^{*}=t_{0}\left(m^{*} / \kappa^{2}\right)$, where $a_{0}, H_{a}$ and $t_{0}$ are the usual atomic units of length, energy, and time, respectively. The value of $\kappa$ is $13.18 \epsilon_{0}$ for GaAs.

In the presence of an external electric pulse, the previous Hamiltonian must be supplemented with a time-dependent term, and the system is governed by the time-dependent Schrödinger's equation during a time interval $[0, \mathrm{~T}]$ :

$$
i \frac{\partial}{\partial t} \Psi(\boldsymbol{r}, t)=\hat{H}(t) \Psi(\boldsymbol{r}, t)=\left[\hat{H}_{0}-\hat{\boldsymbol{\mu}} \boldsymbol{\epsilon}(t)\right] \Psi(\boldsymbol{r}, t),
$$

where the electron-field interaction assumes the dipole approximation in the length gauge, being $\hat{\boldsymbol{\mu}}=-e \hat{\boldsymbol{r}}$ the dipole operator. The time-dependent electric field, $\boldsymbol{\epsilon}(t)=\epsilon(t) \boldsymbol{\pi}$ is linearly polarized in some direction determined by the unit vector $\boldsymbol{\pi}$, contained in the $x y$ plane. The precise direction is in fact irrelevant due to the circular symmetry of the rest of the Hamiltonian. 
The specification of $\epsilon(t)$, together with an initial value condition, determines the full evolution of the system, via the propagation of Schrödinger's equation. The questions that we wish to answer are the following: is it possible, by fine-tuning the form of $\epsilon(t)$, to manipulate at will the spin state of the system in a controllable manner? How fast can this manipulation be performed, as a function of the characteristics of the system - the SOC strength, the presence and magnitude of the external static magnetic field, etc.?

Optimal control is a suitable tool to address this type of questions, reformulating them into the following problem: given a target, defined as the maximization of a functional of the final state of the system (or of its evolution), what is the time-dependent control function that best accomplishes it? In our case, the target must obviously be related to the spin state of the system, whereas the control function is the time-dependent electric field $\epsilon(t)$. The set of possible control functions is the search space for the optimization algorithm. In practice, the control function must be discretized in order to proceed with the numerical computations: a set of parameters $u_{1}, \ldots, u_{M} \equiv \mathbf{u}$ determines the shape of the function: $\epsilon(t)=\epsilon[\mathbf{u}](t)$, and therefore the domain of the parameters $\mathbf{u}$ is the effective search space.

Regarding the target, it is typically defined through the expectation value of some operator $\hat{O}$, i.e. it is a functional of the final state of the system with the form:

$$
F[\Psi]=\langle\Psi(T)|\hat{O}| \Psi(T)\rangle .
$$

Since the parameters $\mathbf{u}$ determine the shape of the control function, which in turns determines the evolution of the system, $\mathbf{u} \rightarrow \Psi[\mathbf{u}]$, the problem is reduced to the maximization of a function of $\mathbf{u}$ :

$$
G[\mathbf{u}]=F[\Psi[\mathbf{u}]]=\langle\Psi[\mathbf{u}](T)|\hat{O}| \Psi[\mathbf{u}](T)\rangle .
$$

This maximization is greatly eased if we have a feasible scheme to compute the gradient of this function, and this is provided by QOCT:

$$
\nabla_{\mathbf{u}} G[\mathbf{u}]=2 \operatorname{Im} \int_{0}^{T} \mathrm{~d} t\left\langle\chi[\mathbf{u}](t)\left|\nabla_{\mathbf{u}} \hat{H}[\mathbf{u}](t)\right| \Psi[\mathbf{u}](t)\right\rangle,
$$

Note that, given the structure of our Hamiltonian:

$$
\nabla_{\mathbf{u}} \hat{H}[\mathbf{u}](t)=(\hat{\boldsymbol{r}} \cdot \boldsymbol{\pi}) \nabla_{\mathbf{u}} \varepsilon[\mathbf{u}](t)
$$

Also note that a new auxiliary wave function $\chi[\mathbf{u}](t)$ has appeared; it is defined as the solution of:

$$
i \frac{\partial}{\partial t} \chi[\mathbf{u}](\boldsymbol{r}, t)=\hat{H}^{\dagger}[\mathbf{u}](t) \chi[\mathbf{u}](\boldsymbol{r}, t), \text { and }
$$




$$
\chi[\mathbf{u}](\boldsymbol{r}, T)=\hat{O} \Psi[\mathbf{u}](\mathbf{r}, T) .
$$

These equations are similar to the equations for the true wave function $\Psi[\mathbf{u}]$, except for the fact that the boundary condition (Eq. (8b)) is given at the final time $t=T$, which implies that $\chi[\mathbf{u}]$ must be propagated backwards. Therefore, the computation of the gradient of $G$, that requires of both wave functions, is computed by first propagating Eq. (3) forward in time and then Eq. (8a) backward. Finally, the maxima of $G$ are found at the critical points $\nabla_{\mathbf{u}} G=0$; in order to find these maxima we use the quasi-Newton method designed by Broyden, Fletcher, Goldfarb and Shanno [58].

It remains to specify the target operator, and the parameterization of the control functions. Regarding the former, we consider two types of targets:

Target A: The goal is to maximize the spin projection onto some direction $\boldsymbol{\xi}$, i.e. the operator $\hat{O}$ is defined as:

$$
\hat{O}=\boldsymbol{\xi} \cdot \hat{\boldsymbol{\sigma}}=\xi_{x} \hat{\sigma}_{x}+\xi_{y} \hat{\sigma}_{y}+\xi_{z} \hat{\sigma}_{z}
$$

For example, if $\xi_{z}=1$, and $\xi_{x}=\xi_{y}=0$ the goal is to maximize the $z$ spin projection. The functional $F$ would therefore be defined as follows:

$$
F[\Psi]=\langle\Psi(T)|\hat{O}| \Psi(T)\rangle=\sum_{i=x, y, z} \xi_{i}\left\langle\Psi(T)\left|\hat{\sigma}_{i}\right| \Psi(T)\right\rangle
$$

Target B: The goal is to populate some selected excited state $\Phi_{f}$, that has the required spin orientation. The target operator is then defined as the projection onto that state:

$$
\hat{O}=\left|\Phi_{f}\right\rangle\left\langle\Phi_{f}\right|
$$

In this case, the functional $F$ is:

$$
F[\Psi]=\langle\Psi(T)|\hat{O}| \Psi(T)\rangle=\left|\left\langle\Phi_{f} \mid \Psi(T)\right\rangle\right|^{2}
$$

Finally, regarding the parametrization of the control function, we expand it first in a Fourier series, and then we enforce several physical constraints: The zero-frequency component is assumed to be zero (in order to ensure that the signal over the full propagation time integrates to zero), and the sum of all the cosine coefficients is also set to zero (in order to 
(a) $\mathrm{B}=0 \mathrm{~T}$

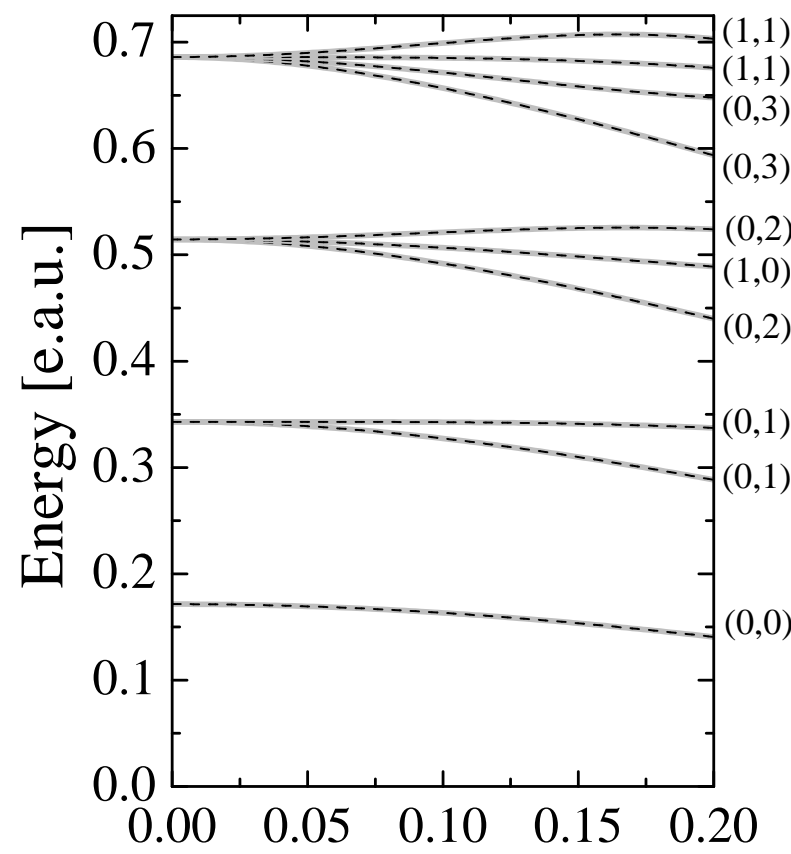

(b) $\mathrm{B}=0.5 \mathrm{~T}$

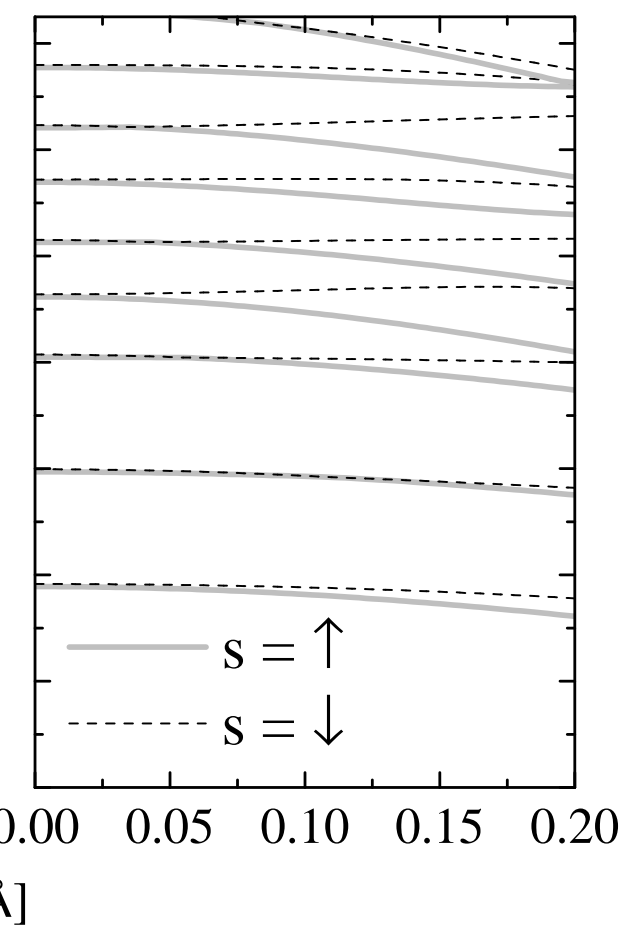

FIG. 1: Energy levels of the parabolic QD versus the strength of the Rashba SOC, $\alpha$, at (a) zero magnetic field and (b) $B=0.5 \mathrm{~T}$. We have labeled the energy levels in (a) as $(n,|l|)$.

ensure that the field will starts and ends at zero). In addition, we enforce a fixed fluence condition:

$$
\int_{0}^{T} \mathrm{~d} t \epsilon^{2}[\mathbf{u}](t)=F_{0} .
$$

The idea is to find the optimal field within the set of fields with equal integrated intensity - this is the physical meaning of the fluence. The set of parameters $\mathbf{u}$ is constructed by considering first the coefficients of the Fourier expansion, and them enforcing the mentioned constraints - for details, see Ref. [55].

\section{NUMERICAL RESULTS AND ANALYSIS}

\section{A. Effect of Rashba SOC and magnetic field on the electronic structure}

To start, we briefly review the effects of SOC on the eigenstates of the QD. In the absence of this SOC, and of any magnetic field, the problem determined by the Hamiltonian 
(a) $\alpha=0$ eV $\AA$



(b) $\alpha=0.2 \mathrm{eV} \AA$

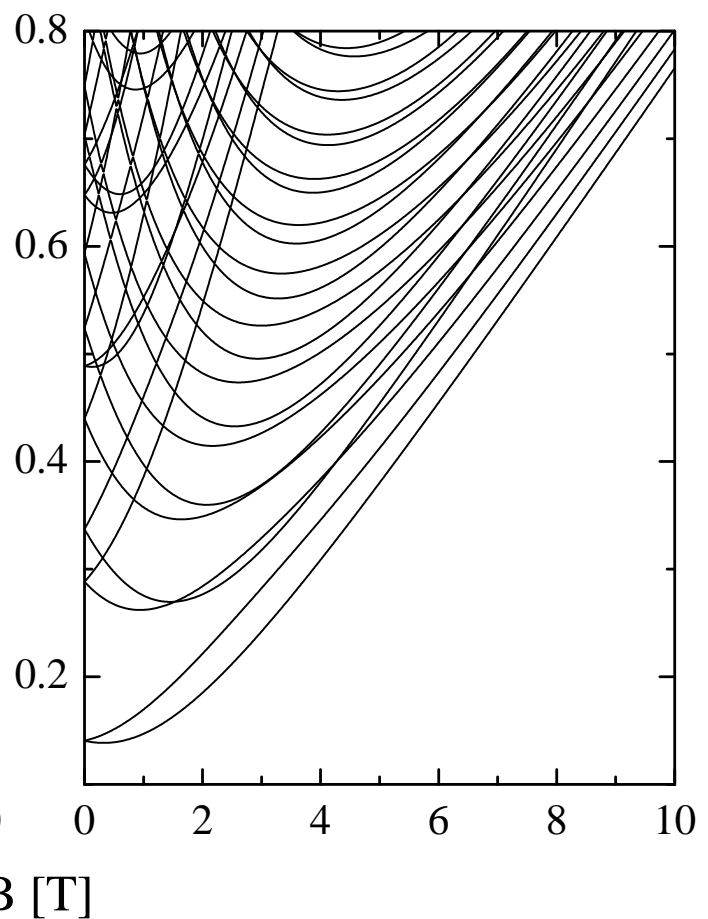

FIG. 2: Fock-Darwin spectrum of the parabolic QD with (a) $\alpha=0$ and (b) $\alpha=0.2 \mathrm{eV} \AA$.

of Eq. (1) is simply a 2D harmonic oscillator. Therefore, the eigenstates are characterized by a principal quantum number $n$, that spans a degenerate subspace of states that differ by their orbital quantum number $l$, and their spin orientation, $s=\uparrow, \downarrow$. In presence of the SOC, the picture changes: Fig. 1 shows the QD electronic energy levels as a function of $\alpha$ at (a) $B=0$ and (b) $B=0.5 \mathrm{~T}$. In (a), the states are labeled by their $(n, l)$ numbers, which are still good quantum numbers. In this zero magnetic field case, the electronic levels undergo an energy shift due to the SOC. This displacement is proportional to $\alpha^{2}$. The SOC also lifts the degeneracy of the electronic states with the same orbital momenta (those with equal $|l|$ ). The separation between these previously degenerate states is also proportional to $\alpha^{2}$. Furthermore, at $B=0$, the energy levels remain doubly degenerate in spin (Kramer's degeneracy).

When a magnetic field is present $(B=0.5 \mathrm{~T}$ [Fig. $1(\mathrm{~b})])$, the usual Zeeman splitting appears. But in addition to this, we can observe the effect of the SOC: an $\alpha$-dependent spin splitting. As we increase the Rashba SOC strength, this splitting gives raise to an approach of energy levels with opposite magnetic moments. Note, however, that due to the SOC, the 
electronic states are no longer pure spin spin-up and spin-down states (in other words, $s$ is no more a good quantum number). Therefore, the labeling $s=\uparrow, \downarrow$ in Figs. 1(a) and 1(b) must be understood only in terms of the spin-branches of each electronic state.

Next, Fig. 2 shows the Fock-Darwin spectra without Rashba SOC and with $\alpha=0.2 \mathrm{eVA}$. It is clear in Fig. 2(b) that the SOC affects the dependence of the electronic states with the magnetic field in comparison with Fig. 2(a), lifting spin degeneracy even at vanishingly small magnetic field. In addition, new crossings of several of the energy levels at low magnetic field regime appears, as well as anti-crossings at higher magnetic field strengths. These anticrossings occur between neighbouring quantum levels with opposite magnetic moments [57].

In the following sections, we will analyze the optimization calculations. As described above, these optimizations are iterative algorithms, and must depart from an initial electric pulse. In all the cases discussed below, we start considering a "reference" pulse of the form:

$$
\epsilon_{\mathrm{ref}}(t)=\epsilon_{0} \cos \left(\omega_{0} t\right) \cos \left(\frac{\pi}{2} \frac{2 t-T}{T}\right)
$$

The peak amplitude $\epsilon_{0}$ is always set to $0.1 \mathrm{kV} / \mathrm{cm}(\sim 0.29$ e.a.u.). Nevertheless, in order to study the effect of initial conditions on the optimization, on each case we have performed four optimization runs starting from four different (random) initial laser pulses, with the fluence [Eq. (13)] obtained from the reference pulse and being preserved by the optimization procedure. The results shown below correspond to the best outcomes. The pulse lengths, given by $T$, will be given in units of $\pi / \omega_{0} \sim 1.15$ ps. The pulses are then represented in a Fourier series, with the constraints discussed above. One of them must obviously be the establishemnt of a cut-off frequency. For all the cases concerning target type A, this cut-off frequency has been set to $\omega_{\text {cut-off }}=20(2 \pi / T)$, which implies 38 degrees of freedom (the number of parameters). For target $B$, we have worked with $\omega_{\text {cut-off }}=10(2 \pi / T)(18$ degrees of freedom).

In the ground state, the expectation value of $\sigma_{z}$ is positive (if no magnetic field is present, the ground state is two-fold degenerate in spin, and then we choose the branch with positive $\left.\left\langle\sigma_{z}\right\rangle\right)$. The goal that we want to achieve is to reverse this spin component. For that purpose, when using a target of type $\mathrm{A}$, we set $\xi_{z}=-1$, and $\xi_{x}=\xi_{y}=0$. For target $\mathrm{B}$, we chose an eigenstate, $\Phi_{f}$, whose $\left\langle\sigma_{z}\right\rangle$ component has opposite sign to that of the ground state, $\Phi_{i}$. 




FIG. 3: (Color on-line) Average spin components (in units of $\hbar$ ) as a function of time for two optimized laser pulses of different lengths, $\pi / \omega_{0} \sim 1.15$ ps and $2 \pi / \omega_{0} \sim 2.3$ ps. We have considered two Rashba SOC strengths: $\alpha=0.05 \mathrm{eV} \AA$ (Figs. (a) and (b)) and $\alpha=0.15 \mathrm{eV} \AA$ (Figs. (c) and (d)).

\section{B. Target A: spin rotation at zero magnetic field}

Fig. 3 shows the time evolution of the average spin components for two optimized laser pulse lengths: the left panels $3(\mathrm{a})$ and $3(\mathrm{c})$ display shorter pulses $\left(T=\pi / \omega_{0}\right)$, and the right panels correspond to double pulse lengths. On the other hand, the top panels 3(a) and 3(b) correspond to a weaker SOC strength, $\alpha=0.05 \mathrm{eV} \AA$, whereas the bottom panels 3(c) and $3(\mathrm{~d})$ correspond to a stronger $\alpha=0.15 \mathrm{eVA}$. If we compare the shorter pulses first ((a) and (c)) it becomes evident how the increased strength of the Rashba SOC results in a spin orientation closer to the target at the end of the pulse - yet this optimization is still not significant. An increase in the pulse lengths, however, results in a very good final outcome even for $\alpha=0.05 \mathrm{eVA}$. In fact, as it can be seen in Fig. 3(d), note that the component $\left\langle\sigma_{z}\right\rangle$ oscillates close to $-1 / 2$ even before the end of the pulse. This tells us that, for that value 


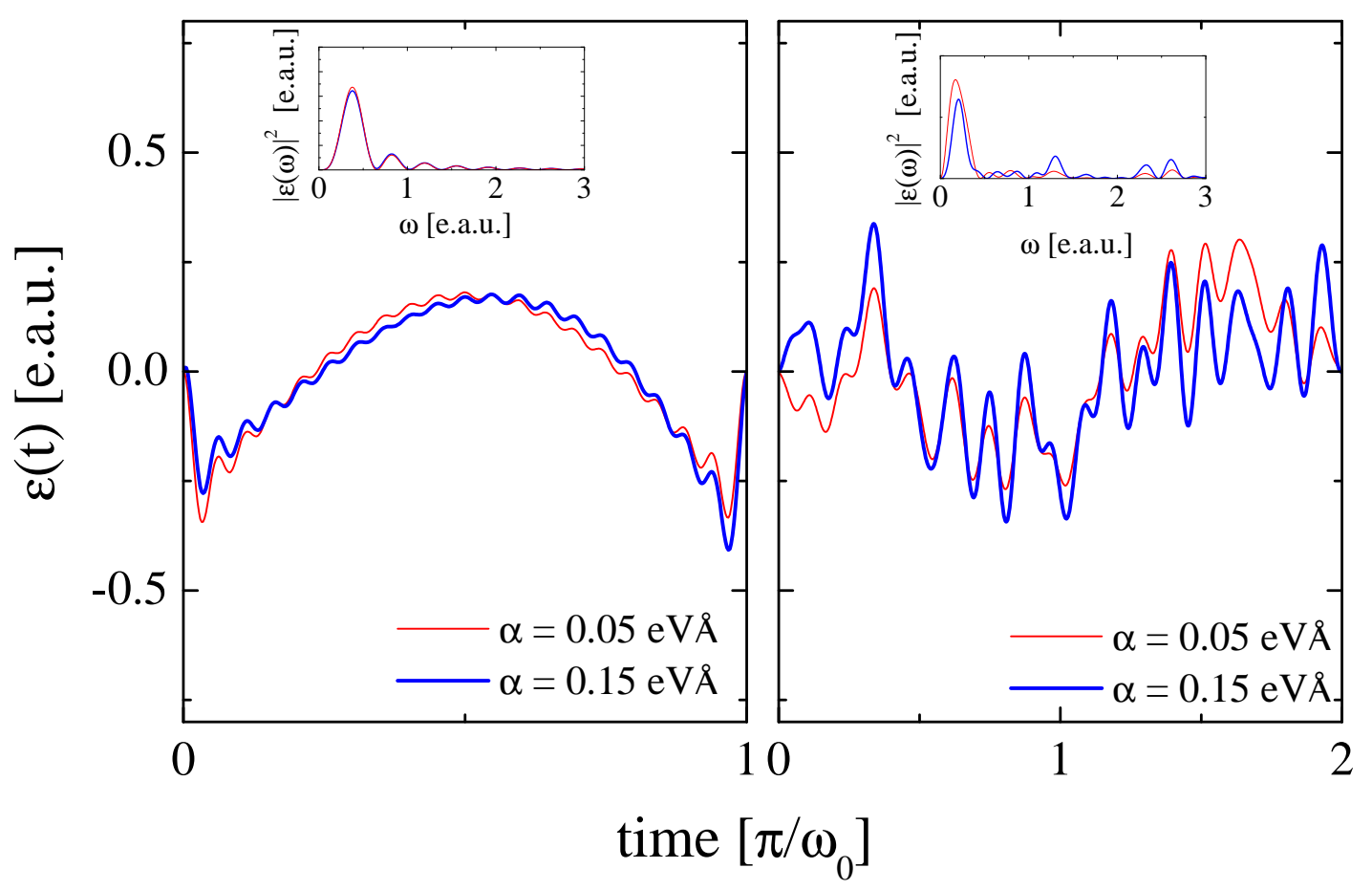

FIG. 4: (Color on-line) Optimal laser pulses corresponding to Fig. 3.

of the SOC strength, an even shorter laser pulse duration would suffice to reach a good $\left\langle\sigma_{z}\right\rangle$ value.

In Fig. 4 we show the optimal laser pulses that produce the results of Fig. 3. Note the very different aspect of the fields needed to optimize the shorter and longer pulses - the latter having a more complex structure. We also display in the inset of each figure the power spectrum of the pulses. The optimal pulses obtained with weaker and stronger SOC do not differ significantly in shape, both in real time and in the frequency domain.

\section{Target A: spin rotation at non-zero magnetic field}

We now consider laser pulse optimizations in the presence of an external magnetic field of $B=0.5 \mathrm{~T}$. Fig. 5 shows the results for a pulse of length $\pi / \omega_{0}$ and four different Rashba SOC strengths. The external magnetic field competes now with the "effective" magnetic field associated to the Rashba SOC and forces the ground state to have a $\left\langle\sigma_{z}\right\rangle$ closer to $1 / 2$. This is evident by looking at the value of $\left\langle\sigma_{z}\right\rangle$ at the initial time in the figure, compared 


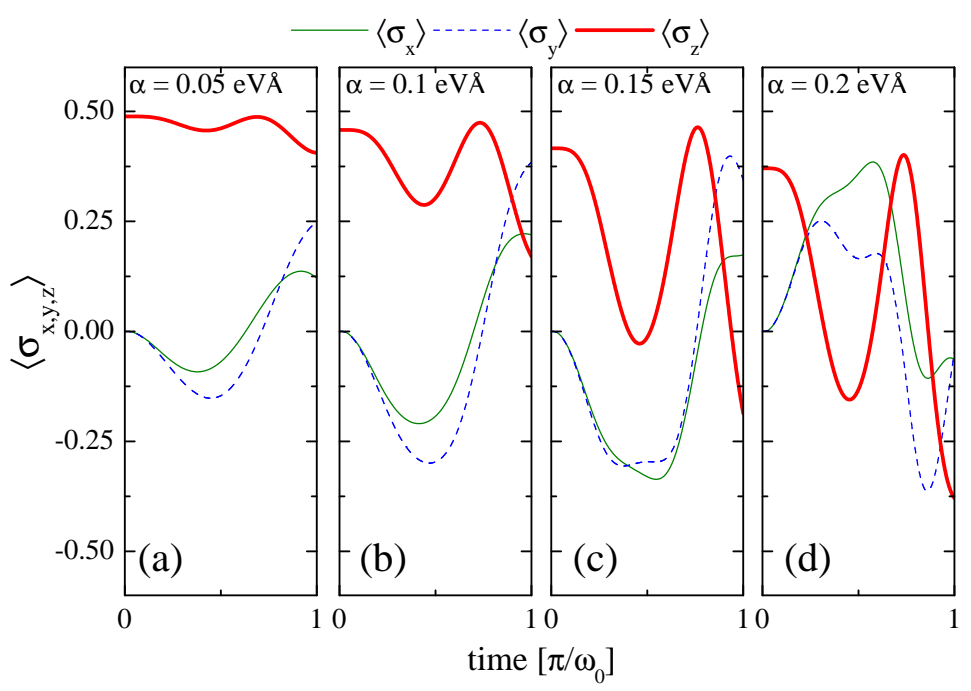

FIG. 5: (Color on-line) Average spin components as a function of time for an optimized laser pulse of length $\pi / \omega_{0}$, with $B=0.5 \mathrm{~T}$ and four Rashba SOC strengths: (a) $\alpha=0.05 \mathrm{eV} \AA$, (b) $\alpha=0.1$ $\mathrm{eVA},(\mathrm{c}) \alpha=0.15 \mathrm{eV} \AA$ and $(\mathrm{d}) \alpha=0.2 \mathrm{eV} \AA$

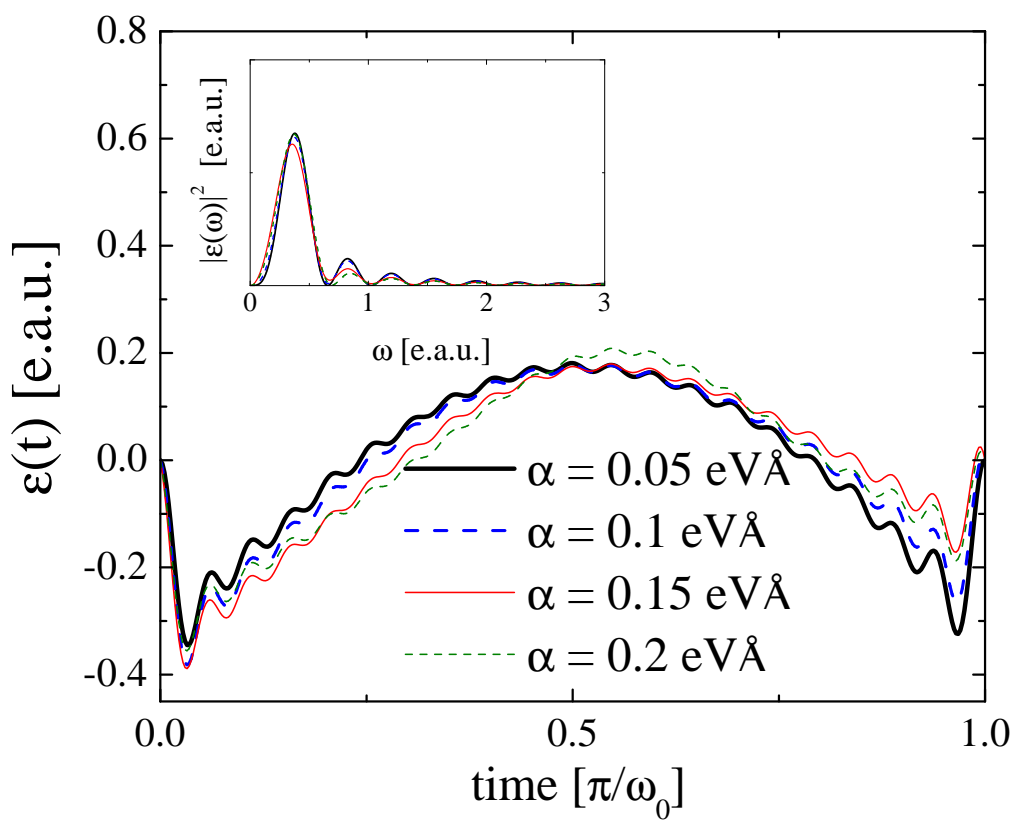

FIG. 6: (Color on-line) Optimized laser pulses corresponding to Fig. 5.

with the initial values of the previous section. We can also observe that increasing the Rashba SOC allows to improve the spin rotation, getting a result very close to the target for $\alpha=0.20 \mathrm{eV} \AA$. Note that, due to the presence of the magnetic field, the starting point is farther from the target, compared to the cases with zero magnetic field, which means that 


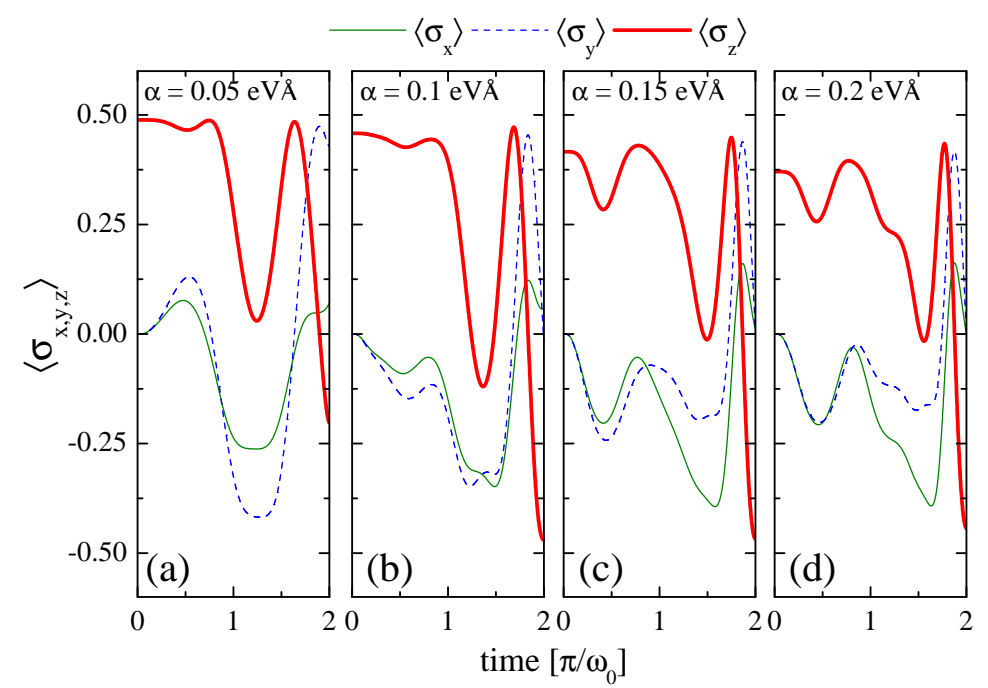

FIG. 7: (Color on-line) Average spin components as a function of time for an optimized laser pulse of length $\pi / \omega_{0}$, with $B=0.5 \mathrm{~T}$ and four Rashba SOC strengths: (a) $\alpha=0.05 \mathrm{eV} \AA$, (b) $\alpha=0.1$ $\mathrm{eVA}$, (c) $\alpha=0.15 \mathrm{eVA}$ and (d) $\alpha=0.2 \mathrm{eV} \AA$.

the spin state must perform a longer path. Fig. 6 shows the optimal pulses associated to Fig. 5. They are qualitatively similar to those shown in Fig. 4.

Let us now consider pulse lengths of $2 \pi / \omega_{0}$ (Fig. 7). In this case, notice that the optimization algorithm is capable of finding pulses that reach the target with smaller SOC strengths than those required with shorter pulses. Regarding the optimal pulses (Fig. 8), note that these look very different to the shorter pulses of length $\pi / \omega_{0}$. One can see how the increase of the SOC strength produces an apparent increase in the number of oscillations of the pulse envelope. In the inset of this figure, note how this increase of the SOC strength results in a splitting of the initial single band into two separate bands. The lower frequency band is associated with the oscillations of the envelope and moves to higher frequency than the initial band as we increase the strength of the SOC.

Finally, Fig. 9 shows the expectation value of $\sigma_{z}$, at the end of the pulse, as a function of $\alpha$ for the two pulse lengths considered. Here it becomes evident how the increase of pulse length allows for a better result. It is also evident that one may get a faster spin-flip through the tuning (increase) of the Rashba SOC. 


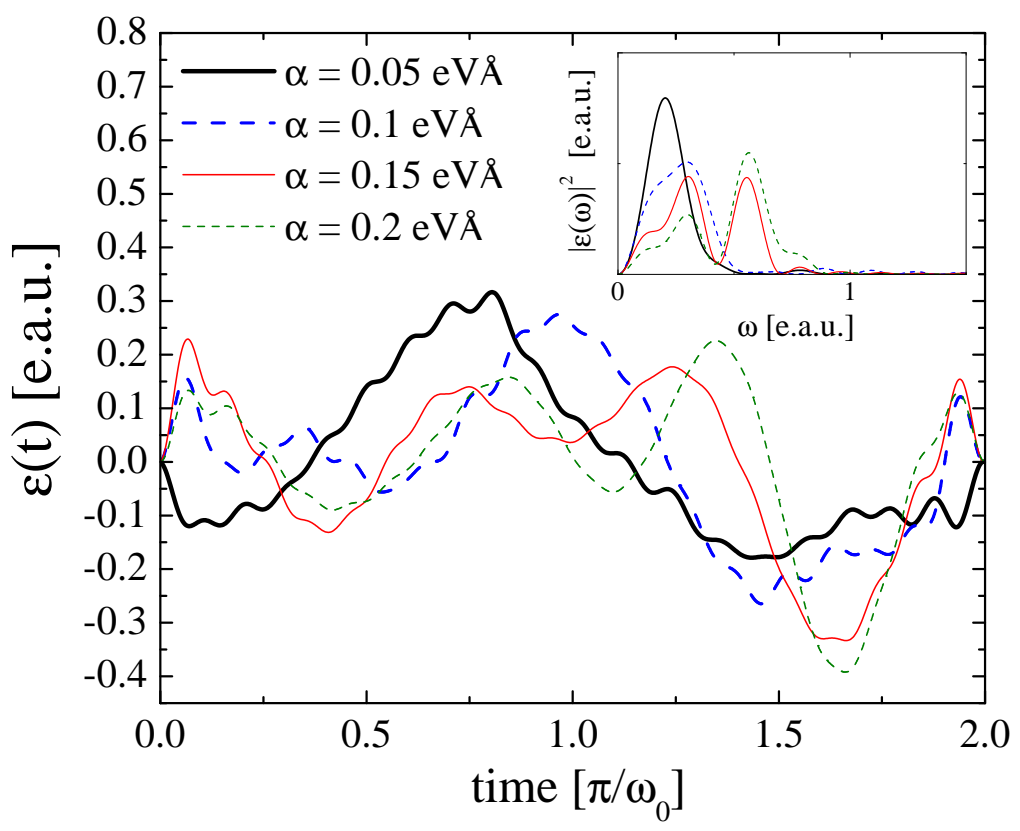

FIG. 8: (Color on-line) Optimized laser pulses corresponding to Fig. 7.

\section{Target B: spin rotation at non-zero magnetic field}

We now describe the results obtained when using a target of type B. We have chosen to maximize the transition between the two spin branches of the ground state, i.e., $E_{0,0, \uparrow} \rightarrow$ $E_{0,0, \downarrow}$ (in the following, the figures will label these two states as $i=0$ and $i=1$, respectively). This transition is only allowed when the two branches are split by the effect of a magnetic field, and therefore, for this calculation, we have set the magnetic field and Rashba SOC strengths as $0.5 \mathrm{~T}$ and $0.2 \mathrm{eV} \AA$, respectively. The cutoff frequency, set in this case as $10\left(2 \pi / \omega_{0}\right)$, is well above the resonant frequency associated with this transition, $\omega_{0 \rightarrow 1}$.

Fig. 10(a) shows the time evolution of the occupation of the first 12 eigenstates of the QD in response to an optimized laser field of length $60\left(\pi / \omega_{0}\right)$. The occupation of state $i=1$ reaches a maximum occupancy of $\sim 0.98$ at the end of the pulse, and the occupation of the ground state decays to zero quickly. During the course of the pulse, the global occupation of those 12 first eigenstates decays almost to zero several times, indicating that in these time intervals the electron is occupying higher energy states. Below, Fig. 10(b) shows the time evolution of $\left\langle\sigma_{x}\right\rangle,\left\langle\sigma_{y}\right\rangle$ and $\left\langle\sigma_{z}\right\rangle$. As expected, the final spin orientation of the electron agrees almost completely with the expectation values of the spin components in state $i=1$.

Fig. 10(c) shows the associated optimal laser field. It is important to remember that, in 




FIG. 9: Expectation value of the $z$-component of the spin at $t=T$ as a function of Rashba SOC strength for two laser pulse lengths.

the case of target $B$, we attempt a state-to-state transition of frequency $\omega_{0 \rightarrow 1}$, indicated by a vertical thick gray line in the inset of Fig. 10(c). In general, despite the random random character of the initial guess field of the search, the optimal pulse is characterized by the presence of a wide distribution of frequencies around $\omega_{0 \rightarrow 1}$.

This is of course not surprising. However, the optimal pulse is not merely a quasi monochromatic pulse with the transition frequency. We have in fact attempted the optimization by starting from these type of pulses, finding that the optimization alters that starting point by adding the necessary extra frequencies to obtain a significantly better result. These runs (not shown here), provided better solutions than the runs started from purely random pulses. Finally, we note that the complexity of the transition process found by the QOCT procedure is also evidenced from the population of many eigenstates during the evolution, far from the two-state model that is used to explain Rabi oscillations. This population of "auxiliary" states is of course also present when using target A, a fact to be expected since the states in these case are not "controlled". Indeed, when using target A even the final state is composed of a superposition of a large number of eigenstates. 


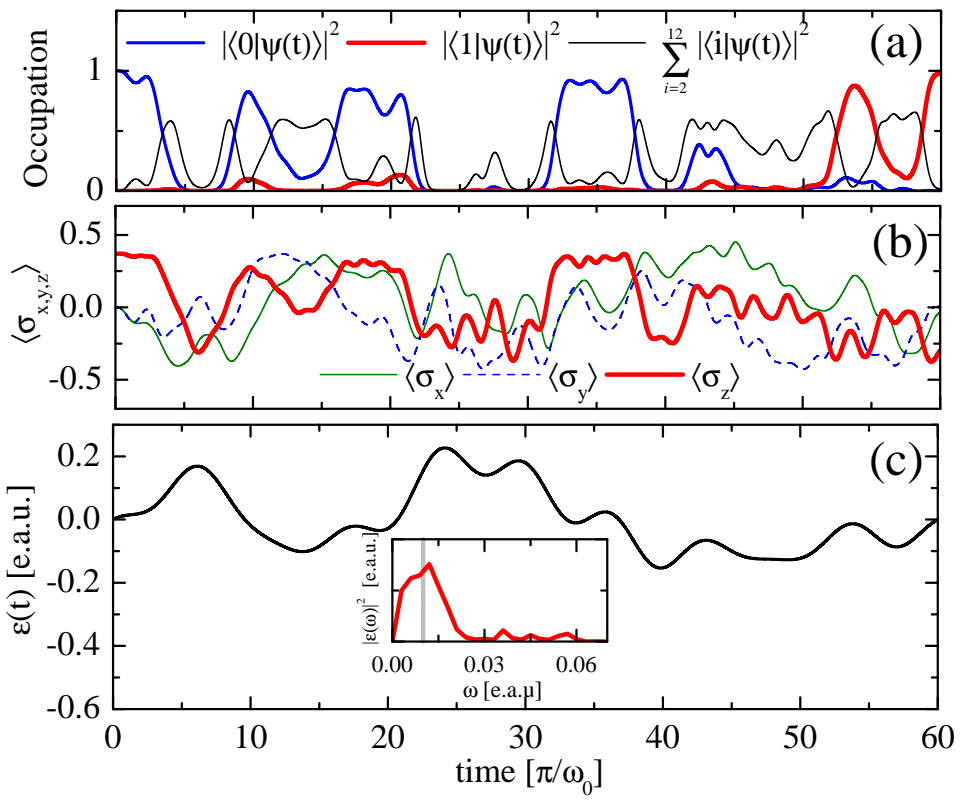

FIG. 10: (Color on-line) (a) Occupation of the 12 first eigenstates as a function of time, (b) average spin components as a function of time and (c) Optimized laser pulse. The length of the pulse is $60\left(\pi / \omega_{0}\right), B=0.5 \mathrm{~T}$ and $\alpha=0.2 \mathrm{eV} \AA$. The inset in (c) shows the power spectrum in frequency of the optimized laser pulse.

\section{CONCLUSIONS}

We have theoretically demonstrated the possibility of manipulating the electronic spin in a semiconductor QD, by means of ultrashort laser pulses, making use of the spin-orbit coupling. We have explored the time scales necessary to perform spin transformations, making use of optimized laser pulse shapes, found with the help of quantum optimal control theory. These time scales depend on the strength of the spin-orbit coupling, and of the presence or absence of an external magnetic field, helpful to fix a value for the initial spin orientation.

The search for an optimal pulse, within QOCT, can be done in multiple ways, and the first choice to make is the design of a target functional. We have shown results for two types of targets. In the first type, the functional only depends on the spin projection value, without placing explicit restrictions on the number of the QD eigenstates which can participate in the representation of the final state. The results show full spin rotations in about 1 or 2 ps, depending on the value of the spin-orbit coupling. In the second type of target, the 
spin control is achieved indirectly through the control of a particular transition between the ground state and an excited state which has the desired spin orientation. In this case, if the target transition involves one of the lowest excited states, the pulse length must be at least an order of magnitude greater than those used in the case of the first target.

In conclusion, our simulations support the idea of ultrafast manipulations of electronic spin in 2D quantum dots, by means of the electric fields of $\mathrm{THz}$ laser pulses, using spin-orbit coupling to transform the electric signal into a spin rotation.

\section{ACKNOWLEDGEMENTS}

This work was supported by the European Communitys FP7 through the CRONOS project, grant agreement no. 280879 .

1 L. P. Kouwenhoven, D. G. Austing, and S. Tarucha, Rep. Prog. Phys. 64, 701 (2001).

2 R. Hanson, L. P. Kouwenhoven, J. R. Petta, S. Tarucha, and L. M. K. Vandersypen, Rev. Mod. Phys. 79, 1217 (2007).

3 I. Žutić, J. Fabian and S. Das Sarma, Rev. Mod. Phys. 76, 323 (2004).

4 Daniel Loss, and David P. DiVincenzo, Phys. Rev. A 57, 120 (1998).

5 J. M. Elzerman, R. Hanson, L. H. Willems van Beveren, B. Witkamp, L. M. K. Vandersypen and L. P. Kouwenhoven, Nature 430, 431 (2004).

6 J. M. Taylor, J. R. Petta, A. C. Johnson, A. Yacoby, C. M. Marcus, and M. D. Lukin, Phys. Rev.B 76, 035315 (2007).

7 D. P. DiVincenzo, Fortschr. Phys. 48, 771 (2000).

8 K. C. Nowak, F. H. L. Koppens, Yu. V. Nazarov and L. M. K. Vandersypen, Science 318, 1430 (2007)

9 P. Stano, and J. Fabian, Phys. Rev. B 77, 045310 (2008).

10 D. Press, T. D. Ladd, B. Zhang and Y. Yamamoto, Nature 456, 218 (2008).

11 Yue Ban, Xi Chen, E. Ya Sherman and J. G. Muga, Phys. Rev. Lett. 109, 206602 (2012).

12 K. De Greeve, D. Press, P. L. McMahon, and Y. Yamamoto, Rep. Prog. Phys. 76, 092501 (2013). 
13 J. H. Jiang, M. Q. Weng, and M. W. Wu, J. Appl. Phys. 100, 063709 (2006).

14 C. Brif, R. Chakrabarti, and H. Rabitz, New J. Phys. 12, 075008 (2010).

15 J. Werschnik, E. K. U. Gross, J. Phys. B 40, R175 (2007).

16 , M. A. L. Marques, A. Castro, G. F. Bertsch, and A. Rubio, Comp. Phys. Comm. 151, 60 (2003).

17 Alberto Castro, Heiko Appel, Micael Oliveira, Carlo A. Rozzi, Xavier Andrade, Florian Lorenzen, M. A. L. Marques, E. K. U. Gross, Angel Rubio, Phys. Stat. Sol. (b) 243, 2465 (2006).

18 X. Andrade, Xavier X. Andrade, J. Alberdi-Rodriguez, D. A. Strubbe, M. J. T. Oliveira, F. Nogueira, A. Castro, J. Muguerza, A. Arruabarrena, S. G. Louie, Alán Aspuru-Guzik, A. Rubio, and Miguel A L Marques, Journal of Physics: Cond. Matt. 24, 233202 (2012).

19 E. Runge and E.K.U. Gross. Phys. Rev. Lett., 52, 997 (1984).

20 Fundamentals of Time-Dependent Density Functional Theory, M. A. L. Marques, N. T. Maitra, Fernando M. S. Nogueira, E. K. U. Gross, and Angel Rubio, editors. Volume 837 of Lecture Notes in Physics. (Springer, Berlin Heidelberg, 2012).

21 E. Räsänen, A. Castro, J. Werschnik, A. Rubio and E. K. U. Gross, Phys. Rev. Lett. 98, 157404 (2007)

${ }^{22}$ E. Räsänen, A. Castro, J. Werschnik, A. Rubio and E. K. U. Gross, Physica E 40, 1593 (2008).

23 E. Räsänen, A. Castro, J. Werschnik, A. Rubio and E. K. U. Gross, Phys. Rev. B 77, 085324 (2008)

24 Y. A. Bychkov and E. Rashba, J. Phys. C 17, 6039 (1984).

25 G. Dresselhaus, Phys. Rev. 100, 580 (1955).

26 P. Pfeffer and W. Zawadzki, Phys. Rev. B 51, 14332 (1995).

27 B. Jusserand, D. Richards, G. Allan, C. Priester and B. Etienne, Phys. Rev. B 51, 4707 (1995).

28 B. Heinrich, Can. J. Phys. 78, 161 (2000).

29 S. A. Wolf, J. Supercond. 13, 195 (2000).

30 S. Das Sarma, J. Fabian, X. D. Hu and I. Žutić, Superlatt. Microstruct. 27, 289 (2000).

31 G. Papp and F. M. Peeters, Appl. Phys. Lett. 78, 2184 (2001).

32 D.C. Ralph and M.D. Stiles, J. Magn. Magn. Matter. 320, 1190 (2008).

33 D. D. Awschalom, D. Loss and N. Samarth, Semiconductor Spintronics and Quantum Computation, Springer, Berlin (2002).

34 J. M. Kikkawa and D. D. Awschalom, Nature 397, 139 (1999). 
See, e.g., D. P. Di Vincenzo and C. H. Bennett, Nature 404, 247 (2000).

36 S. Pancharatnam, Proc. Indian Acad Sci. A 44, 247 (1956).

37 M. I. Dyakonov and V. I. Perel, JETP Lett. 13, 467 (1971).

38 M. V. Berry, Proc. R. Soc. London A 392, 45 (1984).

39 A. G. Aronov, Yu. B. Lyanda-Geller, and G. E. Pikus, Sov. Phys. JETP 73, 537 (1991).

40 N. Nagaosa, J. Phys. Soc. Japan 75, 042001 (2006).

41 B. A. Bernevig, T.L. Hughes, and S.-C. Zhang, Science 314, 1757 (2006).

42 A. P. Peirce, M.A. Dahleh, and H. Rabitz, Phys. Rev. A 37, 4950 (1988)

43 R. Kosloff et al., Chem. Phys. 139, 201 (1989).

44 W. Zhu and H. Rabitz, J. Chem. Phys. 109, 385 (1998).

45 E. Räsänen, A. Castro, J. Werschnik, A. Rubio and E. K. U. Gross, Phys. Rev. Lett. 98, 157404 (2007).

46 E. Räsänen,A. Castro, J. Werschnik, A. Rubio and E. K. U. Gross, Phys. Rev. B 77, 085324 (2008).

47 M. Chen and W. Porod, J. App. Phys. 78, 1050 (1995).

48 S. Lindemann, T. Ihn, T. Heinzel, K. Ensslin, K. Maranowski and A. C. Gossard, Physica E 13, $638(2002)$.

49 J. M. Elzerman, R. Hanson, L. H. Willems van Beveren, L. M. K. Vandersypen and L. P. Kouwenhoven, App. Phys. Lett. 84, 4617 (2004).

50 G. Lommer, F. Malcher, and U. Rössler, Phys. Rev. Lett. 60, 728 (1988).

51 A. P. Peirce, M. A. Dahleh, H. Rabitz, Phys. Rev. A 37, 4950 (1988).

52 R. Kosloff, S. A. Rice, P. Gaspard, S. Tersigni, D. J. Tannor, Chem. Phys. 139, 201 (1989).

53 Y. Ohtsuki, G. Turinici, H. Rabitz, T. J. Chem. Phys. 120, 5509 (2004).

54 I. Serban, J. Werschnik, E. K. U. Gross, Phys. Rev. A 71, 053810 (2005).

55 K. Krieger, A. Castro and E. K. U. Gross, Chem. Phys. 391, 50 (2011)

56 K. Sundermann and R. de Vivie-Riedle, J. Chem. Phys. 110, 1896 (1999).

57 P. Stano and J. Fabian, Phys. Rev. B 72, 155410 (2005).

58 R. Fletcher, Practical Methods of Optimization (Wiley, New York) (2000). 